\title{
Low-Cost, Accessible Fabrication Methods for Microfluidics Research in Low-Resource Settings
}

\author{
Hoang-Tuan Nguyen ${ }^{1}{ }^{\circledR}$, Ha Thach ${ }^{1}$, Emmanuel Roy ${ }^{2}$, Khon Huynh ${ }^{1,+}+(\mathbb{C}$ \\ and Cecile Mong-Tu Perrault ${ }^{3, *,+}$ iD \\ 1 International University—Vietnam National Universities at Ho Chi Minh City, Quarter 6, Linh Trung Ward, \\ Thu Duc District, Ho Chi Minh City 70000, Vietnam; nhtuan@hcmiu.edu.vn (H.-T.N.); \\ hatuny97@gmail.com (H.T.); hckhon@hcmiu.edu.vn (K.H.) \\ 2 Eden Microfluidics, 75011 Paris, France; emmanuel.roy@eden-microfluidics.com \\ 3 Department of Mechanical Engineering, University of Sheffield, Sheffield S1 3JD, UK \\ * Correspondence: c.perrault@sheffield.ac.uk \\ + These authors share senior authorship.
}

Received: 17 August 2018; Accepted: 10 September 2018; Published: 12 September 2018

check for updates

\begin{abstract}
Microfluidics are expected to revolutionize the healthcare industry especially in developing countries since it would bring portable, easy-to-use, self-contained diagnostic devices to places with limited access to healthcare. To date, however, microfluidics has not yet been able to live up to these expectations. One non-negligible factor can be attributed to inaccessible prototyping methods for researchers in low-resource settings who are unable to afford expensive equipment and/or obtain critical reagents and, therefore, unable to engage and contribute to microfluidics research. In this paper, we present methods to create microfluidic devices that reduce initial costs from hundreds of thousands of dollars to about $\$ 6000$ by using readily accessible consumables and inexpensive equipment. By including the scientific community most embedded and aware of the requirements of healthcare in developing countries, microfluidics will be able to increase its reach in the research community and be better informed to provide relevant solutions to global healthcare challenges.
\end{abstract}

Keywords: microfluidics; microfabrication; photolithography; soft lithography; soft embossing

\section{Introduction}

Microfluidics, through its essential role in Lab-on-a-chip (LOC) technologies, plays a key role in the development of rapid, quantitative, and sensitive diagnostic devices. The technology can diminish costs tremendously by reducing both analysis time and the amount of reagents used. Currently, the most compelling application of LOC technologies is in the early and accurate detection of infectious agents in developing countries where resources are severely limited. However, microfluidic research and development is often limited to countries with significant resources for research since current prototyping methods for microfluidic devices are costly and access to materials is limited in low-resource settings [1]. There is often a disconnect between the design of LOC devices and the realities of field application that can be overcome by widening participation in microfluidic prototyping to low-resources setting.

Microfluidics prototyping consists of two main steps: creation of the master mold and replication into a polymeric microfluidic prototype. Master molds are generally created using photolithography, which is a complex and expensive procedure. In the photolithographic process, high-power, collimated ultraviolet (UV) light is projected through a photomask onto a photoresist (SU-8) layer to fabricate patterns [2]. The process is often performed in a clean-room environment, which requires significant capital and maintenance costs. Cheaper alternatives to photolithography include removing the need for 
master molds entirely such as vinyl cutters (xurography) [3,4], laser cutting [5], shrinking polymer [6,7], polyester-toner [8], paper-wax [9], and NOA adhesive [10,11], which have been explored to create master molds and microfluidic chips but lack the superior resolution of the photolithography [1]. Even though 3D printing is also a cheap, robust, and scalable method for producing master mold [12-14], there are still challenges that have prevented the adoption of 3D-printing by microfluidic developers, which are resolution, throughput, and resin biocompatibility $[15,16]$. Attempts to lower the cost of the technique have focused on the UV lighting [17] and alternatives to chrome-plated masks, but the use of a photoresist component, SU-8, has remained constant. However, SU-8 can be prohibitively expensive and complicated to source especially for researchers in low-resource countries. The lack of a high-reproducibility fabrication method that is inexpensive, robust, scalable, and sturdy to use under harsh and resource-limited environments and simple to use is a complication for low-resource countries and prevents them in joining the microfluidics research community.

We explore in this paper an alternative to SU-8 to create a master mold rapidly, cheaply, and with reasonable resolution (100 $\mu \mathrm{m}$ thickness, $100 \mu \mathrm{m}$ feature size) using methacrylate (MA), which exists as a type of nail polish (clear UV gel from Sina ${ }^{\circledR}$, product number: 1940144 , Zhejiang, China). In the early 2000s, methyl(methacrylate) (MMA) was tested as a photo-resistance for microfluidic chips with pre-polymerized MMA placed in a cavity and exposed to UV [18]. However, the volume shrinkage during molding resulted in bubble formation in the PMMA channel plates. Recent development has popularized low-shrinkage meth(acrylates) (MA) first in dental clinics and as furniture sealant and, more recently, as nail polish commonly called "gels." With the expansion of the nail cosmetic market, cheap MA gels and UV lamp sets are now readily available globally and can be exploited to bring microfluidic research in environments previously unable to cater to the high-spec needs of photolithography.

Once the master mold is created, it is used as a template for fabrication of the polymeric device. To date, most microfluidic prototypes are fabricated through soft lithography, which was originally popularized by the Whitesides group [19]. A pre-polymer, most commonly poly(dimethylsiloxane) (PDMS), is cured on top of the master mold and, after curing, a PDMS-negative stamp of the mold is created and bonded irreversibly to glass, which creates the chips. In recent years, it has been recognized that, while PDMS has been widely adopted in academic settings and in a few commercial products (Fluidigm, Cellectricon, etc., it presents significant disadvantages (propensity for protein absorption, difficulties in scaling up for mass production, possible contamination of cyclic silicone monomer derivatives, and reversible hydrophilicity) [20-23] that has limited the translation of prototypes into marketable products [24,25]. Alternatively, a recent thermoplastic (Flexdym ${ }^{\mathrm{TM}}$, Eden Microfluidics, Paris, France) with similar desirable properties to PDMS (optical transparency from 240 to $1100 \mathrm{~nm}$, flexibility, cost-effectiveness, and high patterning fidelity) was presented as an alternative for prototyping that can be easily moved into mass-production. The polymer can be soft embossed on the master mold to replicate its features, can be surface treated for permanent hydrophilicity, and bonded without plasma treatment to glass (bonding strength 1 bar) or polymers (bonding strength up to 3 bars) [26].

In this paper, we report the use of nail polish MA gels and LED-UV light to fabricate microfluidic chips. We demonstrate that the method is able to create master molds with high accuracy and high reproducibility. We also show that the resulting master molds are compatible with two common fabrication methods in microfluidics: soft lithography with PDMS and soft embossing with Flexdym. Overall, the methods used involve minimal capital costs $(<\$ 10,000)$ as well as minimal training and minimal running costs, which makes microfluidics research accessible to a wider community. 


\section{Materials and Methods}

\subsection{Photomask Fabrication}

Photomasks were created using two different printing methods. The first method involves using a 2400 dpi laser printer (Canon LBP3300, Tokyo, Japan) onto a transparent film (Polyethylene terephthalate (PET) A4 transparent sheet), which is commonly used for printed circuit board fabrication. To improve the contrast and homogeneity of the printed pattern, a toner aided spray (Toner Aide Enhancer, Sprayway ${ }^{\circledR}$, Addison, IL, USA) was used by spraying the pattern at a distance of 15 to $20 \mathrm{~cm}$ and dried for $5 \mathrm{~min}$, according to the manufacturer's instructions. This spray dissolves the toner, which enables merging of neighboring ink spots and filling open spaces. This results in darker patterns. The second method involves a widely used printing called offset printing from a printing provider (Saigon 3 Printing Co., Ltd., Ho Chi Minh City, Vietnam).

\subsection{Photolithography Process}

The photolithographic process is described in detail below and in Figure 1.

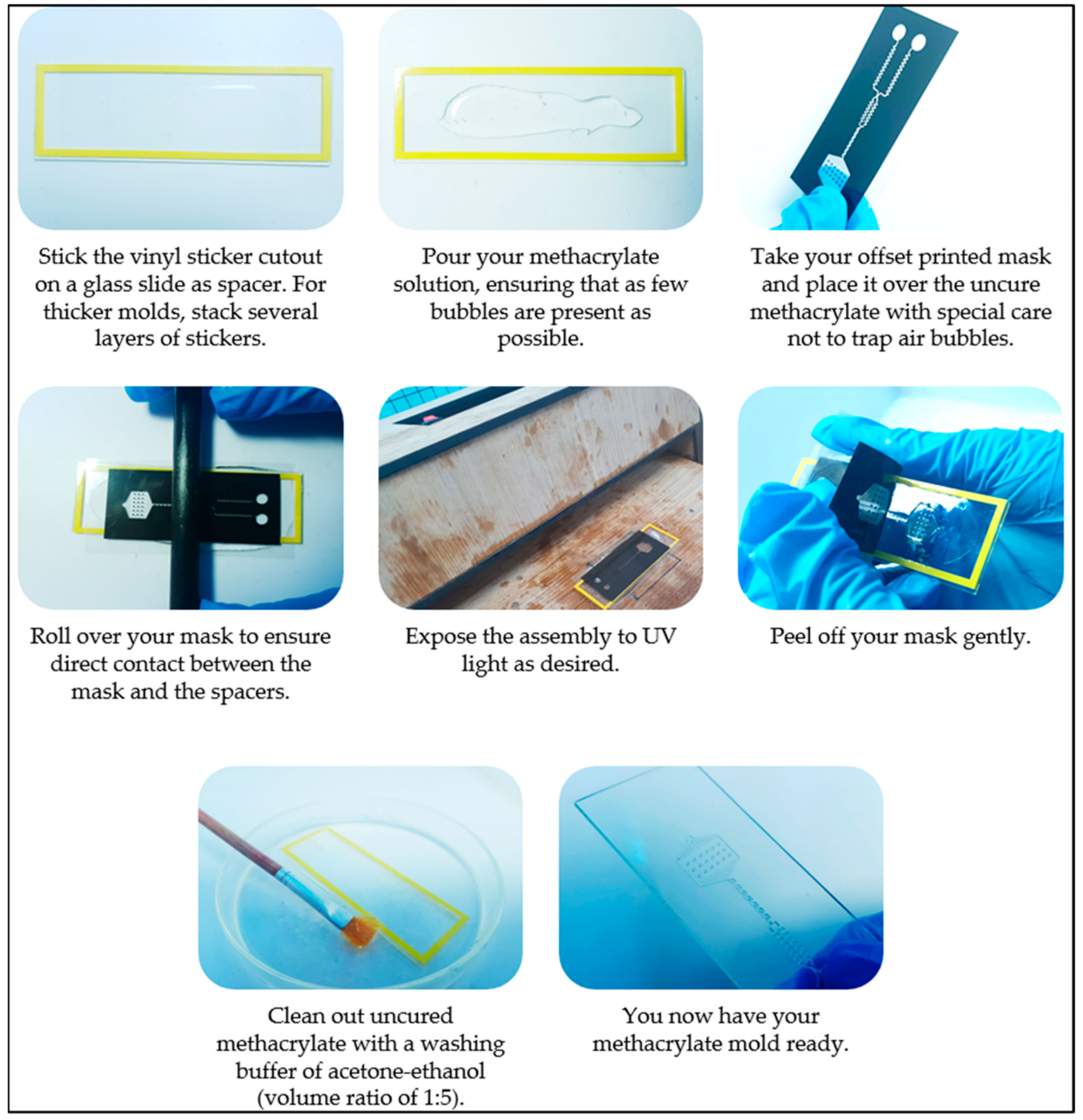

Figure 1. Photolithographic procedure. 


\subsubsection{Methacrylate Coating}

Microscopic glass slides $(25 \mathrm{~mm} \times 75 \mathrm{~mm})$ from Ningbo Greetmed Medical Instruments Co., Ltd. (Ningbo 315040, China) were cleaned using acetone, ethanol, and distilled water. One or several vinyl stickers, $80 \mu \mathrm{m}$ height, were stacked and attached to the glass slides to create a chamber and act as spacers. Approximately $1.2 \mathrm{~mL}$ of methacrylate was placed in the middle of the chamber. The photomask was then placed on top of the chamber in contact with the MA solution. An even spread was ensured by scrolling a rod along the long edge of the chamber.

\subsubsection{UV Exposure}

The methacrylate layer covered with the photomask was exposed under $365 \mathrm{~nm}$ UV light from a 30-watt UV LED array of 30 LEDs (\$21, UV Led Light Bead, product number: DRP-30WGH45UV365, OUMURUI, Guangdong, China). The UV LEDs were powered by a switching power supply (model: JC-480-48, JCPOWER ${ }^{\circledR}$, JunChen-Tech, Guangzhou, China) that is capable of converting $220 \mathrm{~V}$ AC to approximately $35 \mathrm{~V}$ DC.

Under UV illumination, the methacrylate crosslinked into a hardened pattern and adhered to the glass slide. The optimized UV LED-Methacrylate distance was found to be $3 \mathrm{~cm}$ in length and have a methacrylate thickness of $80 \mu \mathrm{m}$ and an illumination time of 15 to $25 \mathrm{~s}$.

\subsubsection{Washing}

After UV illumination, the photomask was peeled off and discarded. The UV exposed methacrylate layer was washed several times with a washing buffer of acetone-ethanol (volume ratio of 1:5) to remove the uncured methacrylate. For sophisticated patterns, the glass slide was dipped into the washing buffer and a paintbrush was used to gently wipe the uncured methacrylate away. Lastly, the cleaned mold was placed at $100^{\circ} \mathrm{C}$ for a few minutes for drying.

\subsection{Molding Microfluidic Chips}

To prove the compatibility of our master molds to create microfluidic prototypes, we tested them with two fabrication methods: soft lithography and soft embossing.

\subsubsection{Soft Lithography}

The PDMS channels were fabricated using Sylgard ${ }^{\circledR} 184$ Silicone Elastomer kit (Dow Corning, Midland, MI, USA). A 10:1 (w/w) ratio of the base and curing agent were thoroughly mixed together and poured over the methacrylate mold, which had been placed inside a Petri dish. The mixture was then degassed in a vacuum desiccator before curing the PDMS for $1 \mathrm{~h}$ at $90^{\circ} \mathrm{C}$. The cured PDMS was then gently peeled off the MA gels mold.

PDMS slabs were bonded with microscope slides after air plasma treatment (evacuating for $30 \mathrm{~s}$, 40-s at high RF power (18 W)) by manually pressing the two parts together).

\subsubsection{Soft Embossing}

Soft embossing was performed using the Flexdym ${ }^{\mathrm{TM}}$ and Sublym $100^{\mathrm{TM}}$ (Eden Microfluidics, Paris, France) system. Flexdym ${ }^{\mathrm{TM}}$ is an alternative material to PDMS with similar mechanical and optical properties. It has the advantage of using conventional manufacturing processes to be molded (hot embossing, extrusion) [26], which permits an easy transition from prototyping to mass production of microfluidic devices. In addition, it offers better hydrophilic properties and easy bonding to most surfaces [26].

A 1-mm thick sheet of Flexdym was placed in contact with the master mold and moved into the Sublym $100^{\mathrm{TM}}$. The system was set to 10 to $12 \mathrm{~min}$ and $105^{\circ} \mathrm{C}$. Once the molding was done, the Flexdym channels were peeled off and bonded to another Flexdym sheet by simple contact pressure. 


\section{Results}

\subsection{Alternative to the Photomask}

In developed countries, photomasks made of chrome-plated glass can be purchased from suppliers. For photomasks with feature sizes over $10 \mu \mathrm{m}$, the cost ranges from \$100-500 depending on the masks' size and material. There is also another option, which is the film photomask provided by companies such as Micro Lithography Services LTD with the cost only about $\$ 100$ for features down to $5 \mu \mathrm{m}$. In Vietnam, no photomasks suppliers could be identified. We, thus, explored photomask alternatives using methods that are readily available in a resource-limited country: printing on a transparency film using an office laser printer and offset printing (at a cost of \$1 per sheet with approximately 20 photomasks).

The preciseness and resolution of the mask are critical to the quality of the lithography. As shown in Figure 2A, the mask printed using the office laser printer is not homogeneous and the edges of the pattern are coarse [27]. This is due to the printing method in which microdots of electrically-charged powder are dispensed and spaced apart to form the image on the film. The gaps let the UV light pass by leasing to an inhomogeneous curing of the methacrylate and rough channel walls. By using a toner aided spray to enhance the contrast of the photomask (Figure 2B), the small gaps can be filled, but the edges are still irregular, which leads to rough channel walls. With the offset printing technique (Figure 2C), the edges of the pattern are smooth and the printed surface is uniformly dark. The offset printing technique sequentially uses four plates with four colors (cyan, magenta, yellow, and black) and a rubber blanket to imprint the image onto the film, which results in a higher quality in the image transfer. UV curing using an offset-printed photomask resulted in channels with sharp walls and uniform cross-linked methacrylate.

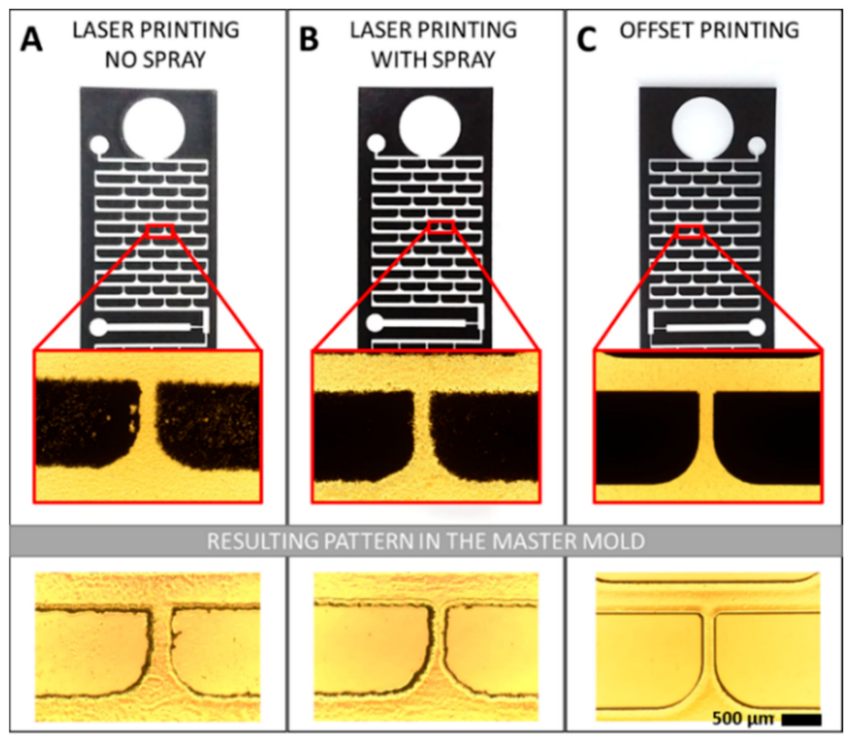

Figure 2. Photomasks were created using printing methods readily available for low-resource research environments. (A) Laser printing from an office printer presents a rough edge. (B) Laser printed masks sprayed a solvent-based solution are more regular but still present coarse walls and overall roughness.

(C) Offset printing showed sharp edges and high contrasts suitable for use as a photomask.

The use of an offset printed transparency represents an excellent alternative to glass photomasks in the low-resources environment.

\subsection{Alternative to the Mask Aligner and Hg-Vapor Lamp}

To overcome the lack of access to photolithography facilities, we explored the use of a LED array. In addition to their wide availability, reasonable cost, and reduced safeguards, there are several 
other advantages in using an LED array over an Hg-vapor lamp including the short rise time to maximum optical intensity $(<300 \mathrm{~ms})$ and low electrical power consumption $(<6 \mathrm{~W})$ [28]. The low power requirement allows the system to run on AA batteries instead of a high-voltage power supply, which is a feature that further enhances its accessibility for low-resource settings.

Since the methacrylate manufacturer did not provide specific information on the energy intensity required for cross-linking, we measured the intensity of the UV light at various distances directly under the light source (Figure 3) to link it with crosslinking performance. We found that, for a $500 \mu \mathrm{m}$ thick layer of methacrylate, a distance of $3 \mathrm{~cm}$ from the UV light resulted in a good reproduction of the pattern design (Figure 3) without over-curing or under-curing, which corresponds to an energy intensity of $18.5 \mathrm{~mW} / \mathrm{cm}^{2}$.

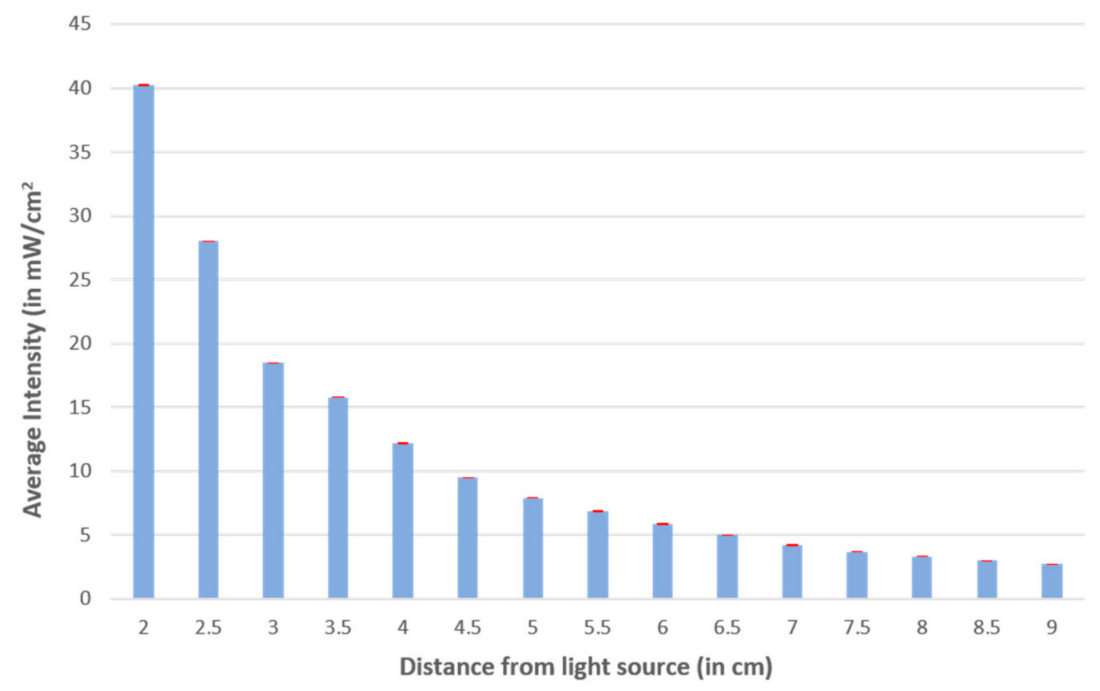

Figure 3. Intensity measurement in $\mathrm{mW} / \mathrm{cm}^{2}$ of the exposure unit at various $\mathrm{y}$ distances from the source.

Since the array of light is not equipped to generate parallel light beams, we explored its impact on wall perpendicularity. We adjusted the distance between the light source and the chip to reduce the divergence angle and create perpendicular sidewalls. We observed a direct correlation between the perpendicular distance between the lamp and the chip and the variation of the wall angle. If the distance was too small $(<1 \mathrm{~cm})$, the entire layer of methacrylate was cured. We found that, for a $500 \mu \mathrm{m}$ thick layer of methacrylate, a distance of $3 \mathrm{~cm}$ from the UV light for $25 \mathrm{~s}$ resulted in perpendicular walls $\left(86.6^{\circ} \pm 1.55^{\circ}\right)$ and no excess uncured methacrylate.

We established the reproducibility of our method by comparing lines of various width from the photomask and that of the resulting MA pattern. For a MA layer of $500 \mu \mathrm{m}$ exposed at $3 \mathrm{~cm}$ from the UV light for $25 \mathrm{~s}$, we found excellent agreement between design width and actual width with a correlation index $\mathrm{R} 2$ of 0.9992 . The ratio between design width and measured width of lines ranging from $150 \mu \mathrm{m}$ to $1000 \mu \mathrm{m}$ was found to be within $1 \pm 0.03$ for all width when measured at the top of the line. When measured from the bottom of the line, the ratio was found to be closer to $0.97 \pm 0.03$ (Figure 4). 


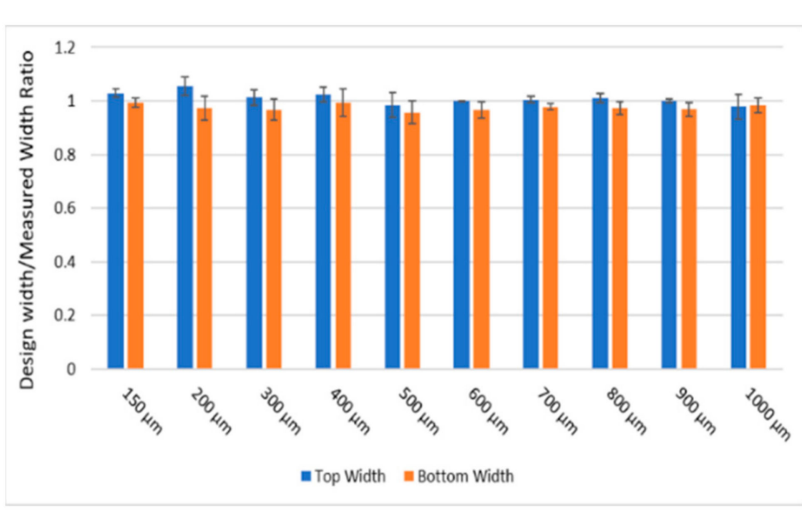

(a)

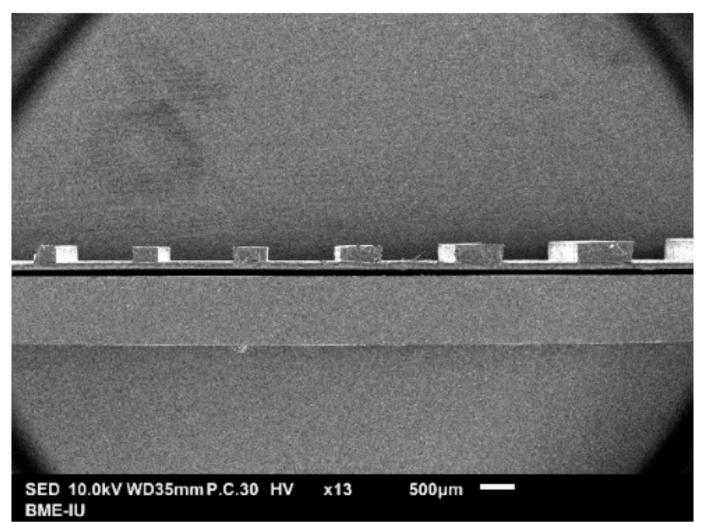

(b)

Figure 4. (a). Ratio between design width and measured width of lines of various width. All lines consisted of $500 \mu \mathrm{m}$ thick layer of methacrylate exposed at a distance of $3 \mathrm{~cm}$ from the UV light for $25 \mathrm{~s}$.

(b). Cross-sectional SEM view of MA lines.

\subsection{Alternative to Spin Coating}

The standard method for controlling the thickness of the photo resistive layer and achieving the micro-channels' height commonly involves a spin coater. For low-resource setting labs that may not be able to afford a spin coater, we found that using vinyl stickers as spacers can be a good alternative method (Figure 5). By changing the height of spacers, we were able to produce microchannels in methacrylate with heights varying from $80 \mu \mathrm{m}$ to $1 \mathrm{~mm}$, which provides a wider range of thicknesses than the conventional photolithographic equivalent using SU-8. However, we noted that, as the layer of methacrylate became thicker, the microfluidics sidewall was no longer perpendicular to the bottom surface. The recorded slope angle created by the sidewall and the bottom surface at $1.3 \mathrm{~mm}$ thick methacrylate (exposed at $3 \mathrm{~cm}$ for $25 \mathrm{~s}$ ) was found to be $115.19^{\circ}$. It is, thus, important to note that the thickness of the gel will influence the final geometry.

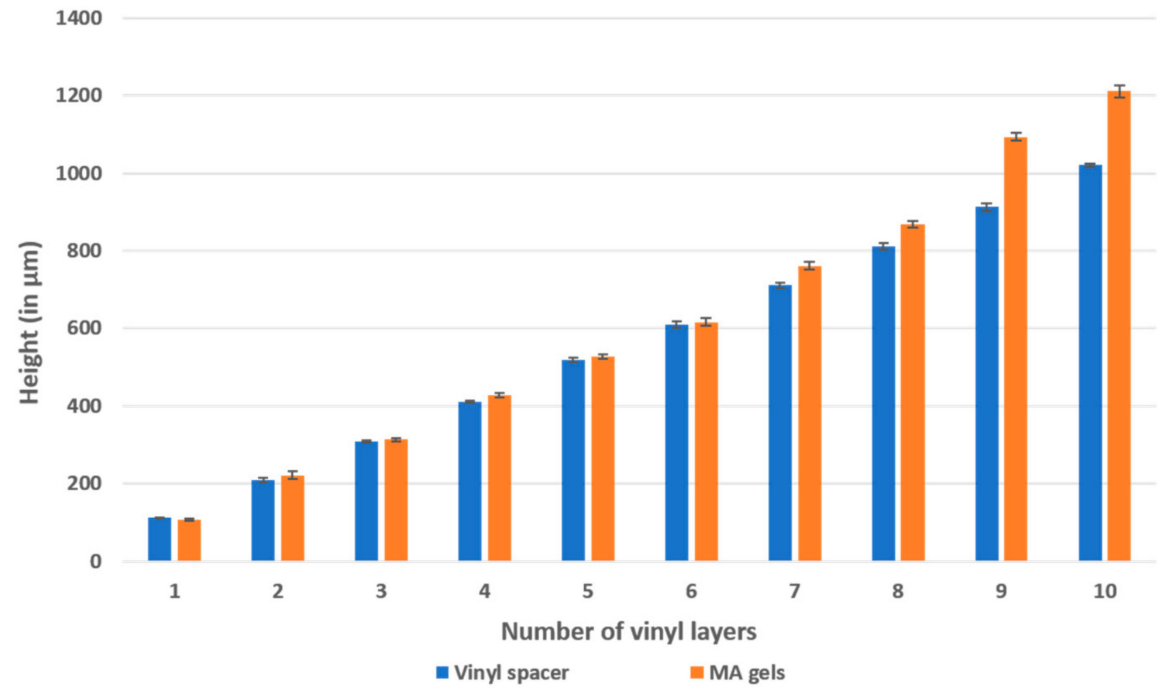

Figure 5. Methacrylate layer thickness according to spacer's height.

\subsection{Compatibility with Microfluidics Fabrication Methods}

The MA molds were used to produce the PDMS chip using soft lithography. The polymer did not adhere to the mold once cured and the patterns were reproduced accurately (Figure 6). The channels were smooth and well-defined, which validates this method for fabrication of microfluidics molds. Figure 6 illustrates several fabricated designs using the proposed method such as capillary pumps and 
gradient generators, which performed satisfactorily and the fluids flowed easily when tested shortly after plasma bonding.

Due to a number of its shortcomings (absorption of small molecules, manufacturing method incompatible with mass production, and reversible hydrophilicity), PDMS is often considered less than ideal for prototyping. We, thus, also tested our master mold with an alternative material, Flexdym, which uses a molding method similar to hot embossing called soft embossing. The method requires less temperature and pressure than the traditional hot embossing method $\left(120^{\circ} \mathrm{C}\right.$ for $5 \mathrm{~min}$ at $\left.5 \mathrm{bar}\right)$. Similar to the PDMS chips, we observed high-reproducibility of the pattern and smooth walls (Figure 6). One additional advantage of the Flexdym material is that bonding does not require plasma treatment, which reduces the initial capital cost by a significant amount. Alternative methods of bonding PDMS without plasma $[10,29,30]$ can also be used, which either adds an adhesive layer to the PDMS or uses stickers while Flexdym present the advantage of an inherently adhesive nature [26]. Lastly, the chips made with Flexdym were more hydrophilic than their PDMS counterpart and, therefore, filled more easily with the reagents.
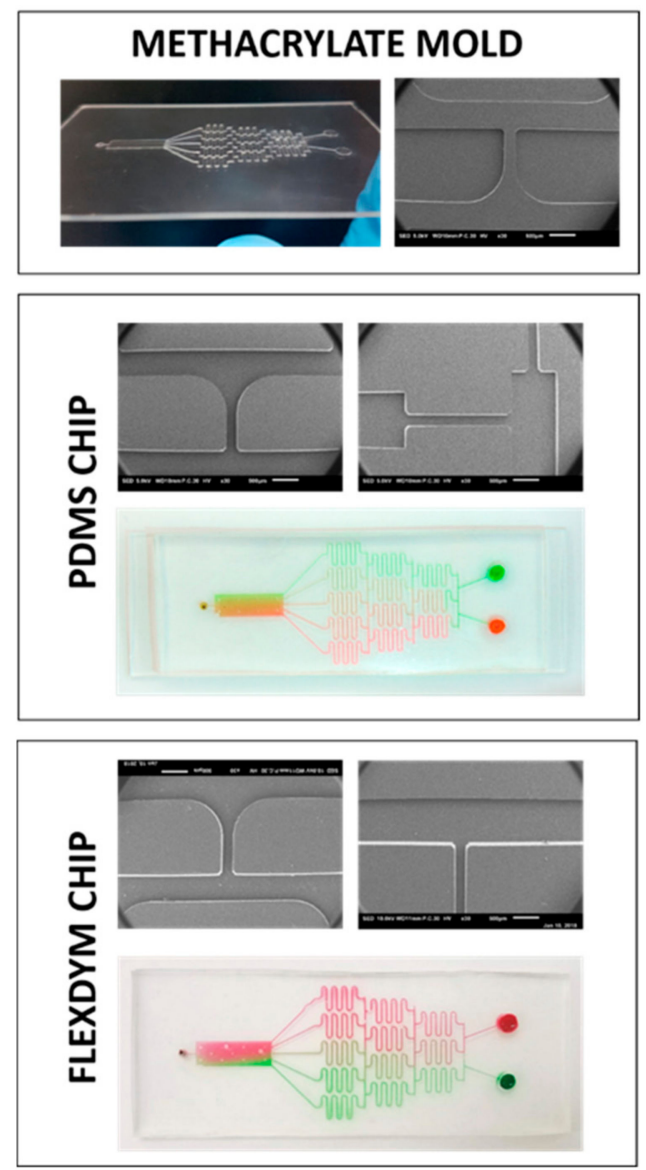

Figure 6. The methacrylate mold was successfully used to reproduce various shapes and design in two polymers adapted to microfluidic applications: PDMS and Flexdym.

\section{Conclusions}

In summary, we have developed an affordable, accessible process to fabricate microfluidic devices using resources available and reasonably-priced for low-resource settings.

This simple process was able to create microfluidic devices with critical feature sizes around $100 \mu \mathrm{m}$ and channel heights up to $1 \mathrm{~mm}$. Importantly, we demonstrated that high-quality patterns could be generated without specialized cleanroom equipment such as mask exposers $(\sim \$ 15 \mathrm{k})$ or aligners $(\sim 50 \mathrm{k})$, spin coaters $(\sim \$ \mathrm{k})$, and plasma machines $(\sim \$ 7 \mathrm{k})$, which lowers the setup cost for a 
microfluidic lab from $\sim \$ 30,000$ to $\$ 65,000$ to about $\$ 6,000$ (UV exposure lamp (\$21) and Sublym100 system $(\sim \$ 6 \mathrm{k}))$. It is important to note that the consumables themselves are massively reduced from an acetate mask ( \$200), SU-8 ( \$400/L), and PDMS ( \$200/kg) to offset printing sheets (\$1/sheet), MA gel $(\$ 10 / 15 \mathrm{~mL})$, and Flexdym $(\sim \$ 25 /$ sheet of $15 \mathrm{~cm} \times 15 \mathrm{~cm})$. We anticipate that the ability to prototype a wide range of devices with minimal setup and running costs will accelerate the development of microfluidic devices and, most importantly, enable researchers from low-resource settings to join the community of microfluidics engineering.

Author Contributions: Supervision-K.H., E.R. and C.M.-T.P.; Designed and performed experiments-H.-T.N. and H.T.; Data analysis-H.-T.N., K.H. and C.M.-T.P.; Writing original draft-all authors; Writing review and editing-K.H. and C.M.-T.P.; Funding acquisition-K.H. and C.M.-T.P.

Funding: This research was funded by EPSRC GCRF Institutional Sponsorship at the University of Sheffield (Project code: X/010734), and Young Science and Technology Incubator Program 2018, Ho Chi Minh city Youth Union's Innovation Centre (Project code: 02/2018/HD-KHCN-VU).

Acknowledgments: The authors are thankful to the Biomedical Engineering Department - International University, Ho Chi Minh city, Vietnam for providing the necessary resources required for this work. We thank BlackHole Lab for providing all information on pricing of equipment for microfabrication.

Conflicts of Interest: The research being reported in this publication was partially supported by Eden Microfluidics. The authors E.R. and C.M.P. of this publication have equity ownership in Eden Microfluidics, which is developing products related to the reported research.

\section{References}

1. Sharma, H.; Nguyen, D.; Chen, A.; Lew, V.; Khine, M. Unconventional low-cost fabrication and patterning techniques for point of care diagnostics. Ann. Biomed. Eng. 2011, 39, 1313-1327. [CrossRef] [PubMed]

2. Nie, Z.; Kumacheva, E. Patterning surfaces with functional polymers. Nat. Mater. 2008, 7, 277-290. [CrossRef] [PubMed]

3. Taylor, D.; Dyer, D.; Lew, V.; Khine, M. Shrink film patterning by craft cutter: complete plastic chips with high resolution/high-aspect ratio channel. Lab Chip 2010, 10, 2472. [CrossRef] [PubMed]

4. Yuen, P.K.; Goral, V.N. Low-cost rapid prototyping of flexible microfluidic devices using a desktop digital craft cutter. Lab Chip 2010, 10, 384-387. [CrossRef] [PubMed]

5. Wang, W.; Zhao, S.; Pan, T. Lab-on-a-print: from a single polymer film to three-dimensional integrated microfluidics. Lab Chip 2009, 9, 1133. [CrossRef] [PubMed]

6. Sollier, K.; Mandon, C.A.; Heyries, K.A.; Blum, L.J.; Marquette, C.A. "Print-n-Shrink" technology for the rapid production of microfluidic chips and protein microarrays. Lab Chip 2009, 9, 3489. [CrossRef] [PubMed]

7. Grimes, A.; Breslauer, D.N.; Long, M.; Pegan, J.; Lee, L.P.; Khine, M. Shrinky-Dink microfluidics: rapid generation of deep and rounded patterns. Lab Chip 2008, 8, 170-172. [CrossRef] [PubMed]

8. Piccin, E.; Ferraro, D.; Sartori, P.; Chiarello, E.; Pierno, M.; Mistura, G. Generation of water-in-oil and oil-in-water microdroplets in polyester-toner microfluidic devices. Sens. Actuators B Chem. 2014, 196, 525-531. [CrossRef]

9. Mao, X.; Huang, T.J. Microfluidic diagnostics for the developing world. Lab Chip 2012, 12, $1412-1416$. [CrossRef] [PubMed]

10. Bartolo, D.; Degré, G.; Nghe, P.; Studer, V. Microfluidic stickers. Lab Chip 2008, 8, 274-279. [CrossRef] [PubMed]

11. Silvestrini, S.; Ferraro, D.; Tóth, T.; Pierno, M.; Carofiglio, T.; Mistura, G.; Maggini, M. Tailoring the wetting properties of thiolene microfluidic materials. Lab Chip 2012, 12, 4041-4043. [CrossRef] [PubMed]

12. Waldbaur, A.; Rapp, H.; Länge, K.; Rapp, B.E. Let there be chip—towards rapid prototyping of microfluidic devices: one-step manufacturing processes. Anal. Methods 2011, 3, 2681. [CrossRef]

13. Kamei, K.; Mashimo, Y.; Koyama, Y.; Fockenberg, C.; Nakashima, M.; Nakajima, M.; Li, J.; Chen, Y. 3D printing of soft lithography mold for rapid production of polydimethylsiloxane-based microfluidic devices for cell stimulation with concentration gradients. Biomed. Microdevices 2015, 17, 36. [CrossRef] [PubMed]

14. Ferraro, D.; Serra, M.; Ferrante, I.; Viovy, J.-L.; Descroix, S. Microfluidic valve with zero dead volume and negligible back-flow for droplets handling. Sens. Actuators B Chem. 2018, 258, 1051-1059. [CrossRef] 
15. Bhattacharjee, N.; Urrios, A.; Kang, S.; Folch, A. The upcoming 3D-printing revolution in microfluidics. Lab Chip 2016, 16, 1720-1742. [CrossRef] [PubMed]

16. Waheed, S.; Cabot, J.M.; Macdonald, N.P.; Lewis, T.; Guijt, R.M.; Paull, B.; Breadmore, M.C. 3D printed microfluidic devices: enablers and barriers. Lab Chip 2016, 16, 1993-2013. [CrossRef] [PubMed]

17. Li, Y.; Wu, P.; Luo, Z.; Ren, Y.; Liao, M.; Feng, L.; Li, Y.; He, L. Rapid fabrication of microfluidic chips based on the simplest LED lithography. J. Micromech. Microeng. 2015, 25, 055020. [CrossRef]

18. Chen, Y.; Zhang, L.; Chen, G. Fabrication, modification, and application of poly(methyl methacrylate) microfluidic chips. Electrophoresis 2008, 29, 1801-1814. [CrossRef] [PubMed]

19. McDonald, J.C.; Duffy, D.C.; Anderson, J.R.; Chiu, D.T.; Wu, H.; Schueller, O.J.A.; Whitesides, G.M. Fabrication of microfluidic systems in poly(dimethylsiloxane). Electrophoresis 2000, 21, 27-40. [CrossRef]

20. Toepke, M.W.; Beebe, D.J. PDMS absorption of small molecules and consequences in microfluidic applications. Lab Chip 2006, 6, 1484-1486. [CrossRef] [PubMed]

21. Wang, J.D.; Douville, N.J.; Takayama, S.; ElSayed, M. Quantitative Analysis of Molecular Absorption into PDMS Microfluidic Channels. Ann. Biomed. Eng. 2012, 40, 1862-1873. [CrossRef] [PubMed]

22. Tsao, C.-W. Polymer Microfluidics: Simple, Low-Cost Fabrication Process Bridging Academic Lab Research to Commercialized Production. Micromachines 2016, 7, 225. [CrossRef]

23. Halldorsson, S.; Lucumi, E.; Gómez-Sjöberg, R.; Fleming, R.M.T. Advantages and challenges of microfluidic cell culture in polydimethylsiloxane devices. Biosens. Bioelectron. 2015, 63, 218-231. [CrossRef] [PubMed]

24. Mukhopadhyay, R. When PDMS isn't the best. Anal. Chem. 2007, 79, 3248-3253. [CrossRef] [PubMed]

25. Gratton, S.E.A.; Williams, S.S.; Napier, M.E.; Pohlhaus, P.D.; Zhou, Z.; Wiles, K.B.; Maynor, B.W.; Shen, C.; Olafsen, T.; Samulski, E.T.; DeSimone, J.M. The Pursuit of a Scalable Nanofabrication Platform for Use in Material and Life Science Applications. Acc. Chem. Res. 2008, 41, 1685-1695. [CrossRef] [PubMed]

26. Lachaux, J.; Alcaine, C.; Gómez-Escoda, B.; Perrault, C.M.; Duplan, D.O.; Wu, P.-Y.J.; Ochoa, I.; Fernandez, L.; Mercier, O.; Coudreuse, D.; Roy, E. Thermoplastic elastomer with advanced hydrophilization and bonding performances for rapid (30 s) and easy molding of microfluidic devices. Lab Chip 2017, 17. [CrossRef] [PubMed]

27. Ahmadzadeh, F.; Tsai, S.S.H.; Papini, M. Effect of curing parameters and configuration on the efficacy of ultraviolet light curing self-adhesive masks used for abrasive jet micro-machining. Precis. Eng. 2017, 49, 354-364. [CrossRef]

28. Huntington, M.D.; Odom, T.W. A Portable, Benchtop Photolithography System Based on a Solid-State Light Source. Small 2011, 7, 3144-3147. [CrossRef] [PubMed]

29. Serra, M.; Pereiro, I.; Yamada, A.; Viovy, J.-L.; Descroix, S.; Ferraro, D. A simple and low-cost chip bonding solution for high pressure, high temperature and biological applications. Lab Chip 2017, 17, 629-634. [CrossRef] [PubMed]

30. Wu, H.; Huang, B.; Zare, R.N. Construction of microfluidic chips using polydimethylsiloxane for adhesive bonding. Lab Chip 2005, 5, 1393. [CrossRef] [PubMed] 